\title{
Sweet syndrome induced by SARS-CoV-2 Pfizer-BioNTech mRNA vaccine
}

Anne-Sophie Darrigade ${ }^{1}$, Hélène Théophile ${ }^{2}$, Paola Sanchez-Pena ${ }^{2}$, Brigitte Milpied ${ }^{1}$, Marianne Colbert ${ }^{3}$, Stéphane Pedeboscq ${ }^{1}$, Thierry Pistone ${ }^{1}$, Marie-Laure Jullie ${ }^{1}$, and Julien Seneschal ${ }^{4}$

${ }^{1} \mathrm{CHU}$ de Bordeaux

${ }^{2} \mathrm{CHU}$ Bordeaux GH Pellegrin

${ }^{3}$ Groupe Bordeaux Nord Aquitaine

${ }^{4}$ Centre Hospitalier Universitaire de Bordeaux

March 12, 2021

\section{Sweet syndrome induced by SARS-CoV-2 Pfizer-BioNTech mRNA vaccine}

AS Darrigade, $\mathrm{MD}^{1}, \mathrm{H}$ Théophile, $\mathrm{MD}^{2}$, $\mathrm{P}$ Sanchez-Pena, $\mathrm{MD}^{2}$, B Milpied, MD ${ }^{1}$, M Colbert ${ }^{4}$, MD, S Pedeboscq $^{5}, \mathrm{MD}, \mathrm{T}$ Pistone ${ }^{6}, \mathrm{MD}, \mathrm{ML}$ Jullié, $\mathrm{MD}^{7}$, J Seneschal, MD, PhD ${ }^{1,3}$

${ }^{1}$ : Department of Adult and Pediatric Dermatology, Bordeaux University Hospitals, France

2 : Department of pharmacovigilancy, Bordeaux University Hospitals, France

3 : Research Unit INSERM U1035

${ }^{4}$ : Department of geriatry, Clinic Bordeaux Nord, Bordeaux, France

5 : Department of pharmacology, Bordeaux University Hospitals, France

${ }^{6}$ : Department of infectious disease, Bordeaux University Hospitals, France

7: Department of anatomopathology, Bordeaux University Hospitals, France

Manuscript word count: 607

Key words : sweet syndrome, SARS-CoV-2, Pfizer-BioNTech mRNA vaccine, delayed hypersensitivity, IDR

Corresponding author: A.S. Darrigade, Dermatology Department, Saint-André Hospital, 1, rue Jean Burguet 33000 Bordeaux, France

Phone: +33556794705

Fax: +33556794975

anne-sophie.darrigade@chu-bordeaux.fr

Funding source: No financial disclosures

Financial Disclosure: No external funding for this manuscript

To the editor,

A 45-year-old woman, without any past medical history or allergy presented in our clinic with a rapid onset of diffuse skin eruptions. Five days earlier, she received the first injection of the SARS-CoV-2 Pfizer-BioNTech 
mRNA. Concomitantly she took $1000 \mathrm{mg}$ paracetamol to prevent any post-vaccination syndrome. She well tolerated the preceding vaccines (influenza every year) before this one.

The eruption started $24 \mathrm{~h}$ after vaccine injection and was composed at time of the clinical exam of erythematous infiltrated papulosis located all over the body, without face involvement (Figure 1). No other extracutaneous symptoms were noted. Blood exams showed increased blood count levels with increased neutrophils count $(8.77 \mathrm{G} / \mathrm{l})$, hepatic cytolysis (AST 67UI/L and ALT 116UI/l) with high level of PCR (115mg/l). SARS-CoV-2 PCR test and serology were negative. Viral tests for EBV, CMV, parvovirus B19, and Herpes simplex/Herpes zoster showed only a slight EBV reactivation. Histopathological examination of the skin biopsy showed a hyperplastic epidermis with an edematous papillary dermis. A superficial and deep dermal perivascular, periadnexal and interstitial dense infiltrate composed of neutrophils, eosinophils and lymphocytes was also a feature. Leukocytoclastic vasculitis was also seen (Figure 2A-2B). Clinical and pathological exams were compatible with the diagnosis of SS induced by SARS-CoV-2 Pfizer-BioNTech mRNA vaccine. Systemic steroid therapy (prednisone $0.5 \mathrm{mg} / \mathrm{kg} / \mathrm{d}$ ) for five days was started and led to rapid improvement of the skin condition without any recurrence after treatment discontinuation. She did not receive the second vaccine injection.

Patch-tests performed (14 days after steroid treatment stop, one month after SS) on both on healed and normal skin with pur SARS-CoV-2 Pfizer-BioNTech mRNA vaccine prepared less than 4 hours before were negative (Figure 1C 2-3). Then, intradermoreaction (IDR) with vaccine diluted at 1/10 on normal skin was positive in delayed reading (Figure $1 \mathrm{C} \mathrm{1}$ ). Cutaneous biopsy was realized on the positive IDR reaction, showing an abundant inflammatory infiltrate predominantly with lymphocytes (Figure 2C).

Cutaneous reactions after vaccine injection are rare, and heterogenous ${ }^{1}$. They could be related to the vaccine or the adjuvant. In addition, vaccine could trigger flares of chronic inflammatory conditions as it was previously reported ${ }^{1}$. At that time, minor local side effects are reported with SARS-CoV-2 vaccines such as pain, swelling or redness; hypersensitivity reactions were anaphylactic reaction but no severe delayed hypersensitivity are reported ${ }^{2-3}$. Three cases of acute febrile neutrophilic dermatosis are reported in the international bank of WHO, one in United Kingdom, one in United States of America and our case. Under SARS-CoV-2 Pfizer-BioNTech mRNA vaccine four cases of vasculitis had been reported after injection. In France one case of relapse of neutrophilic disorder was reported one day after SARS-CoV-2 Pfizer-BioNTech mRNA vaccine. The adjuvant associated with the SARS-CoV-2 Pfizer-BioNTech mRNA vaccine is polyethylene glycol (PEG) $2000^{3}$. However our patient never received infusion containing PEG or polysorbate before. Patchtests with PEG or polysorbate alone were not performed because of the negativity of the patch-test with the SARS-CoV-2 Pfizer-BioNTech mRNA vaccine. Only 10 cases of SS induced by vaccine are published so far including: 3 with seasonal influenza, 1 with influenza A, 2 with pneumococcal, 2 tuberculosis, 2 small pox ${ }^{4}$. SS is an acute inflammatory skin disease associated with important infiltration of neutrophils. Leukocytoclastic vasculitis could be present in $\mathrm{SS}^{5}$. One case of SS in a patient receiving pneumococcal vaccine showed the presence of dermal vasculitis associated with infiltration of neutrophils ${ }^{6}$. In case of anaphylactic reaction under SARS-CoV-2 Pfizer-BioNTech mRNA vaccine, the risk of relapse with the Moderna SARS-CoV-2 mRNA vaccine or SARS-CoV-2 vaccines with an adenovirus carrier and protein subunit remains unknown ${ }^{3}$, in case of SS even more.

To conclude we report the first case of SS induce by SARS-CoV-2 Pfizer-BioNTech mRNA vaccine confirmed by positive IDR.

\section{References}

1. Rosenblatt AE, Stein SL. Cutaneous reactions to vaccinations. Clin Dermatol. 2015;33(3):327-32

2. Castells MC, Phillips EJ. Maintaining Safety with SARS-CoV-2 Vaccines. N Engl J Med. 2021;384(7):643-649.

3. Banerji A, Wickner PG, Saff R, Stone CA Jr, Robinson LB, Long AA, Wolfson AR, Williams P, Khan DA, Phillips E, Blumenthal KG. mRNA Vaccines to Prevent COVID-19 Disease and Reported Allergic Reactions: Current Evidence and Suggested Approach. J Allergy Clin Immunol Pract. 2020:S2213- 
2198(20)31411-2

4. Pedrosa AF, Morais P, Nogueira A, Pardal J, Azevedo F. Sweet's syndrome triggered by pneumococcal vaccination. Cutan Ocul Toxicol. 2013;32(3):260-1.

5. Ratzinger G, Burgdorf W, Zelger BG, Zelger B. Acute febrile neutrophilic dermatosis: a histopathologic study of 31 cases with review of literature. Am J Dermatopathol. 2007;29(2):125-33.

6. Machan M et al. Pegfilgrastim-induced Sweet's syndrome: a case report. Int J Dermatol 2014;53 : $1275-7$

Figures legends:

Figure 1: Sweet syndrome lesions

A- First localization appeared $24 \mathrm{~h}$ after the vaccine injection on the back

B- Erythematous papulosis on the left shoulder

C- 1) Cutaneous tests: Positive IDR with SARS-CoV-2 Pfizer-BioNTech mRNA vaccine diluted to 1/10e at day5

2) Patch test with pur SARS-CoV-2 Pfizer-BioNTech mRNA vaccine in healed skin

3) Patch test with pur SARS-CoV-2 Pfizer-BioNTech mRNA vaccine in normal skin

Figure 2: A- HEx5: papillary dermal edema, interstitial, perivascular and periadnexal superficial and deep dermal polymorphous inflammatory infiltrate

B- HEx20: leucocytoclastic vasculitis, eosinophils (white arrow), perivascular lymphocytes and interstitial neutrophils

C- HEx3: perivascular and periadnexal superficial and deep lymphocytic infiltrate

Acknowledgement:

The authors acknowledge the Uppsala Monitoring Centre (UMC) which provided some data presented in the present study. The opinions and conclusions in this study are not necessarily those of the WHO.
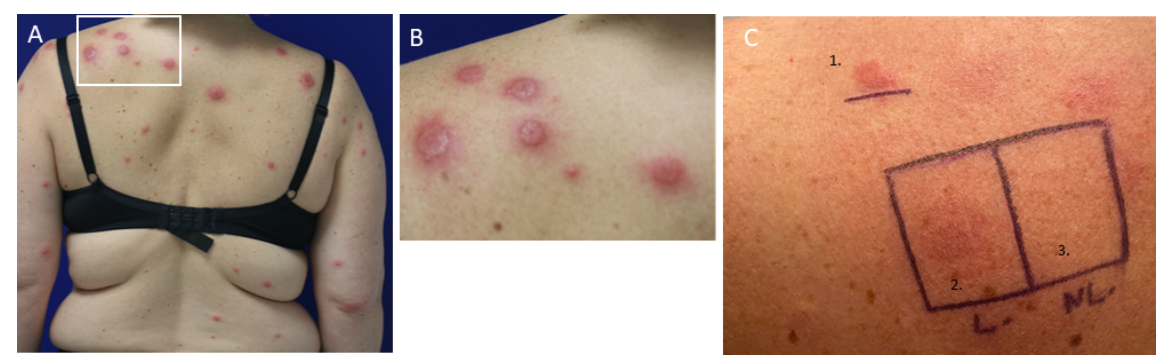

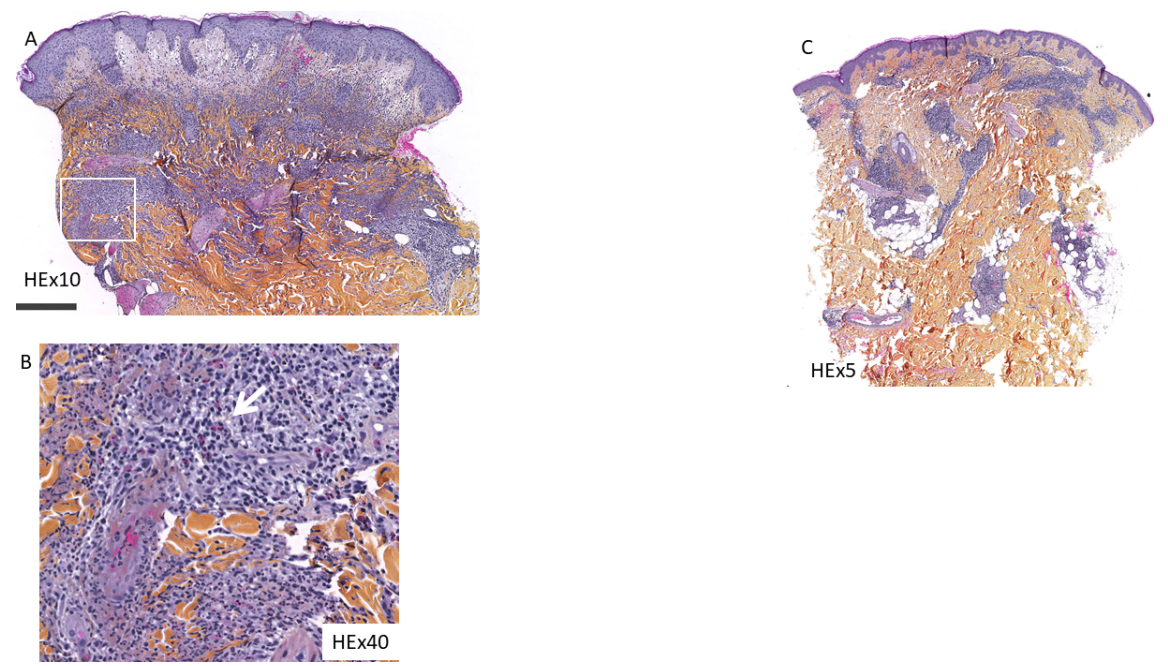